\title{
Fibrous Hamartoma of Infancy at the Hand: A Case Report with Complications
}

\author{
Nicholas Sacksteder ${ }^{\text {1*, Daniel Jones }}{ }^{1}$, David S. Brink², Joao Panattoni1 \\ ${ }^{1}$ Department of Orthopaedic Surgery, Saint Louis University School of Medicine, St. Louis, MO, USA \\ ${ }^{2}$ Department of Pathology and Pediatrics, Saint Louis University School of Medicine, St. Louis, MO, USA \\ Email: sacsktedernj@slu.edu
}

Received 30 January 2015; accepted 23 March 2015; published 26 March 2015

Copyright (C) 2015 by authors and Scientific Research Publishing Inc.

This work is licensed under the Creative Commons Attribution International License (CC BY).

http://creativecommons.org/licenses/by/4.0/

(c) (i) 0 pen Access

\begin{abstract}
Fibrous Hamartoma of Infancy is a benign subdermal tumor most commonly presenting at the proximal appendages. Here we present a case of Fibrous Hamatoma of Infancy at the hand with discussion of its challenging excision and post-operative complications. This case highlights the delicate and challenging nature of pediatric hand surgery.
\end{abstract}

\section{Keywords}

Fibrous Hamartoma, Fibrous Hamartoma of Infancy, Pediatric Hand

\section{Introduction}

Since Reye's six-patient case series in 1956, greater than 200 cases of Fibrous Hamartoma of Infancy have been described in the literature [1]. Here we discuss the case of a two-year-old male presenting with slow growth of two small tumors at the palm of his hand. Pathologic evaluation reveals this lesion to be a hamartoma.

There are five case reports of Fibrous Hamartoma of Infancy (HoI) in the hand at the time of this composition [2]-[6]. Despite appearing superficial and freely mobile on clinical evaluation, the lesions prove to be challenging to dissect due to the depth that they descended into the palm and their adherence to the superficial skin. This emphasizes the importance of having HoI among the differential diagnoses and, more importantly, of being prepared for an operation that may involve deep dissection away from delicate neurovascular structures. This superficial dissection causes some post-operative wound complications that will be discussed further in the present article.

HoIs are characterized by their benign course. They typically reveal themselves between birth and two years

${ }^{*}$ Corresponding author. 
of age. They are predominantly found on the axilla, proximal appendages, groin, and neck regions [7]. They have no clear familial inheritance or environmental trigger, but chromosomal abnormalities are described [4]. The tumors do no undergo metastasis; though, local invasion leads to morbidity.

The purpose of this paper is to demonstrate the difficulty of excision of a clinically straightforward disease process.

\section{Case Report}

Here we present the case of a two-year-old child presenting with six months of painless soft tissue growth at the palm of his left hand. The child was born full term by caesarian section. He was without previous medical diagnosis and surgery. His only medication was certirizine for seasonal allergies.

The child presented to clinic with two spherical lesions at the palmar aspect of the left hand for six months (Figure 1). One was $1.0 \mathrm{~cm}$ round. The other, much smaller, was less than $0.5 \mathrm{~cm}$ round. They were adjacent to each other along the distal palmar crease. The larger lesion appeared first, and, around one month later, the smaller satellite lesion was observed by the parents. The patient offered no pain complaints. The tumors did not functionally interfere with the child's daily activities. The sensation to light touch was normal with no altered skin turgor and normal capillary refill. Considering the masses were increasing in size with the appearance of a satellite lesion, surgical excision was recommended.

The patient was placed supine on the surgical table with the left upper limb on a hand table. The limb was lightly exsanguinated, and a tourniquet was inflated with a pressure of $200 \mathrm{mmHg}$. A 2-cm volar incision was made following the distal palmar crease. The two masses were located and identified as being composed of fibrofatty tissue. The tissue was closely adherent to the palmar skin, with no clear plane of dissection. Therefore, the skin had to be separated from the tumor through a process similar to full thickness skin graft harvesting.

The tumors were observed to be deeper than expected, surrounding the common digital nerve and superficial palmar arch (Figure 2). Due to the unexpected size and depth of the mass, the wound had to be extended proximally in a zig-zag fashion for visualization. A plane of dissection could then been identified between the fatty infiltrate and the neurovascular structures, and the two masses were meticulously excised and send to pathology. The main tumor measured $1.5 \mathrm{~cm} \times 1.0 \mathrm{~cm}$. The smaller round lesion was $0.5 \mathrm{~cm}$ in diameter (Figure 3). The two lesions were not connected.

The wound was closed with 5-0 chromic interrupted simple sutures, and a bulky soft dressing was applied. Five days after the surgery, the parents were instructed to do dressing changes and start with soapy soaks. They found that the wound had partially dehisced (Figure 4). Steri-strips were applied for re-approximation, and an intrinsic-plus short arm cast was placed for protection until healing. The cast was removed two weeks later, and the child was found to have progressed to full healing. There were no functional limitations after the operation, with painless full range of motion and normal sensation (Figure 5).

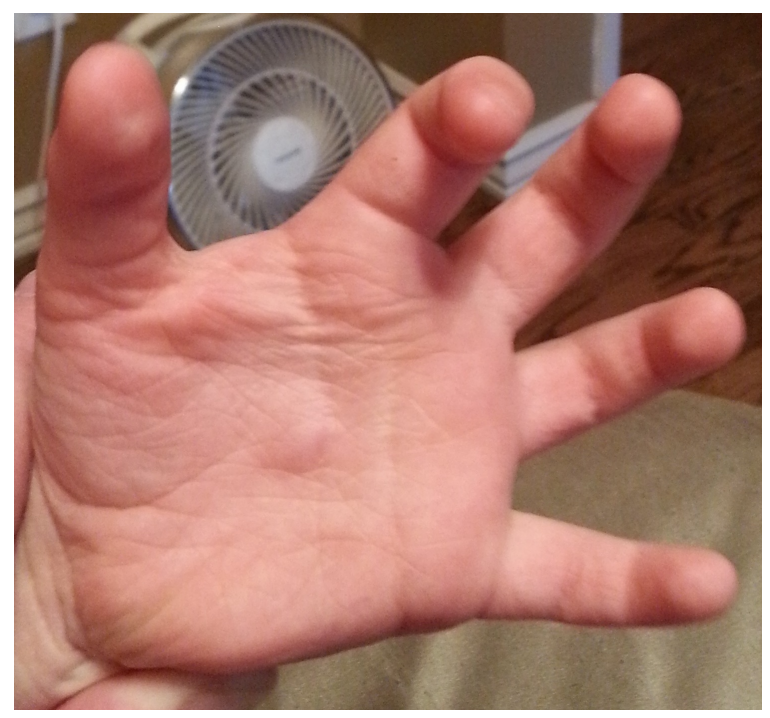

Figure 1. Preoperative photograph of lesions. 


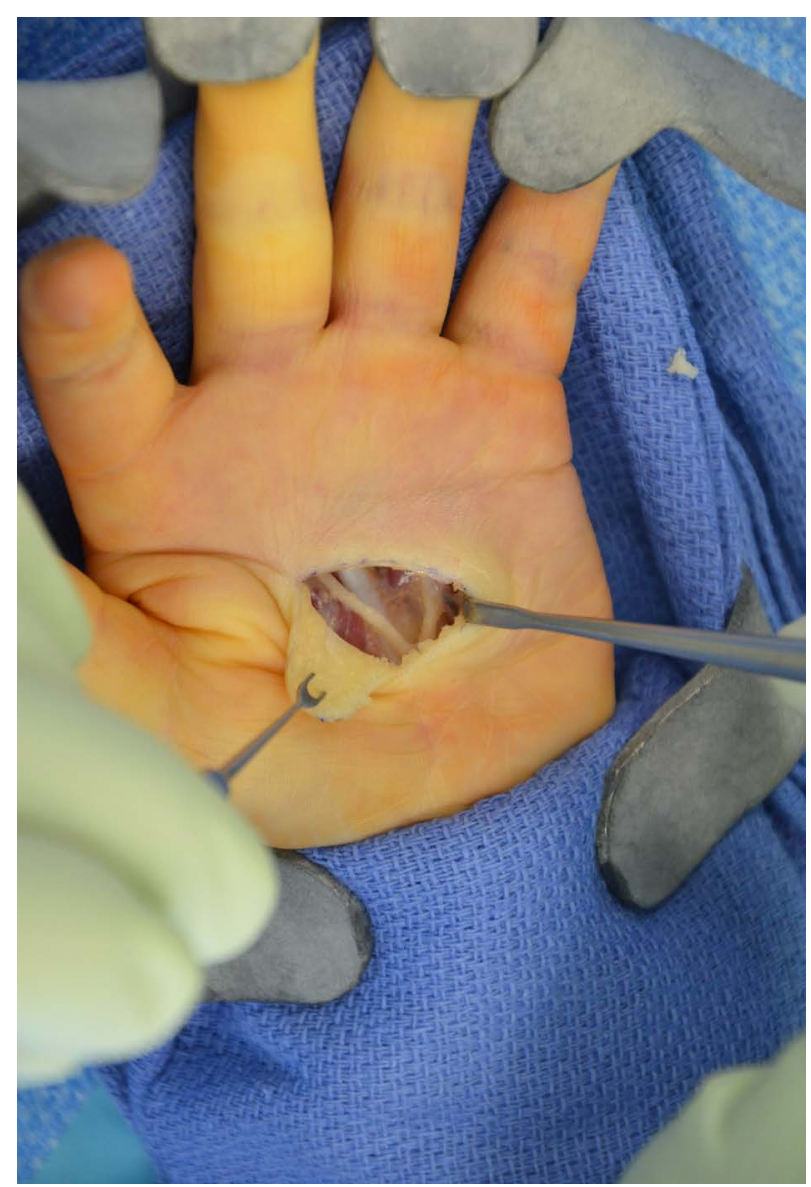

Figure 2. Intraoperative photograph. Note the close relation to the common digital nerve and superficial palmar arch.

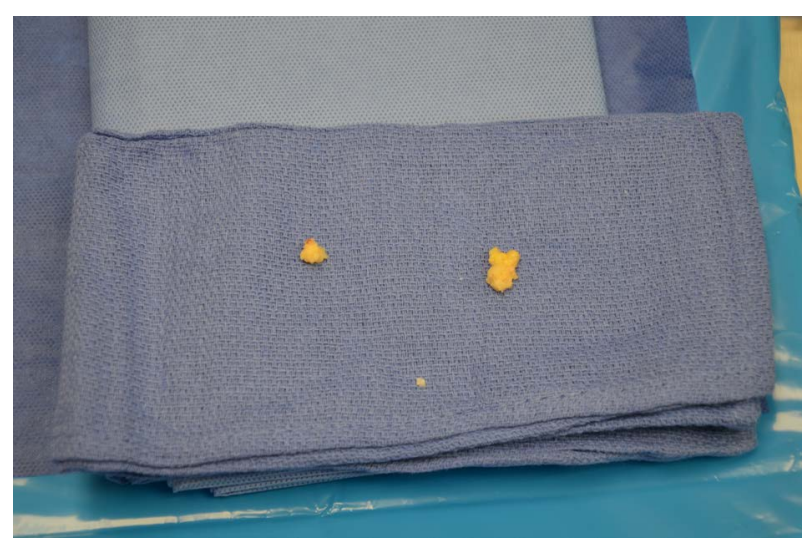

Figure 3. Macroscopic aspect intra-operatively.

Microscopic examination of the tumors showed an organoid distribution of mature adipose tissue; trabeculae of fibrous connective tissue, and nests of loosely arranged spindle cells within Alcian-blue-positive mucoid matrix (Figure 6-8). Immunohistochemical staining showed spindle cell cytoplasm to be diffusely S100-negative and focally actin-positive; spindle cells showed a diffuse negative nuclear label for beta-catenin.

\section{Discussion}

Fibrous Hamartoma of Infancy is a benign yet concerning finding on any child. It typically presents within the 


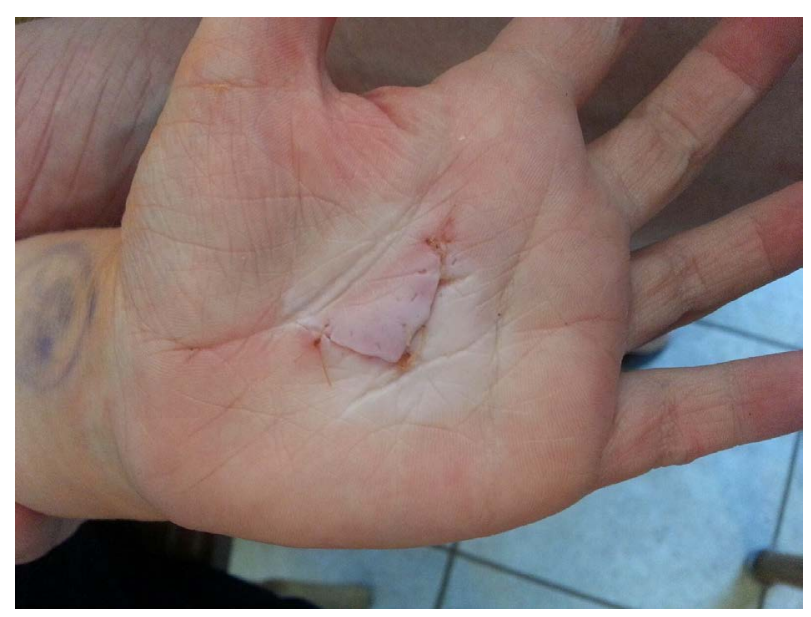

Figure 4. Partial dehiscence, 5 days after surgery.

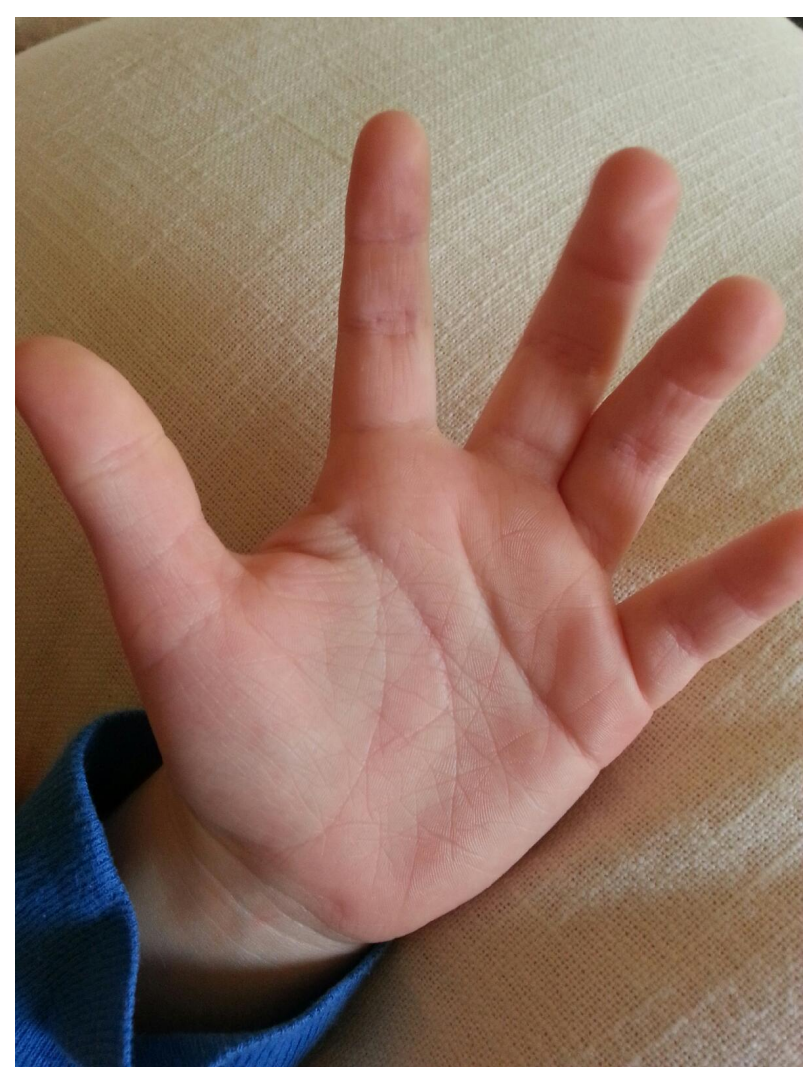

Figure 5. Post-operative images, 6 months after surgery.

first two years of life. Many are present at birth, and the average age of identification is 8 months. They are most commonly located at the groin or axilla as a freely mobile painless lump within the surrounding tissues. They exhibit near linear growth but tend to plateau with time. There is a male to female ratio of 1:2 [7]. No syndromic associations have been identified. Macroscopic changes in the overlying skin have been described but are rare [7]; however, the overlying skin is almost always found to have undergone characteristic changes on microscopic examination [8].

Local excision is most often curative for HoI. Sotelo-Avila et al. [9] suggest a 15\% recurrence rate. Recurrence is thought to be the results of inadequate excision. There are no descriptions of adequate margins in the literature, but reasonable care should be taken for complete excision. Staged excisions are a described treatment strategy [2]. 


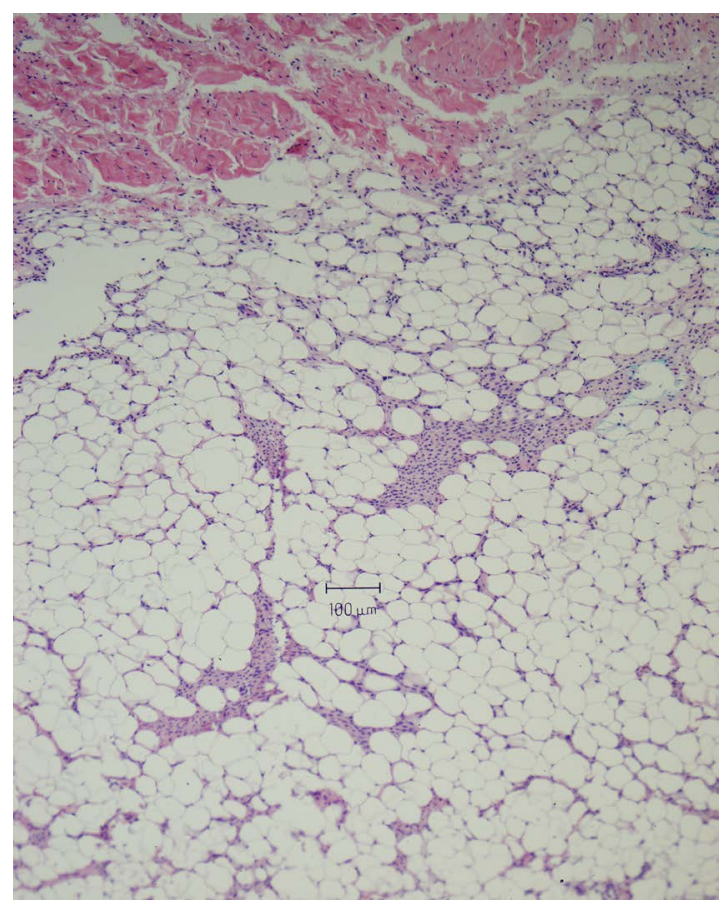

Figure 6. Histologically, the lesion is composed of fibrous connective tissue trabeculae (top quarter of image) and adipose connective tissue (bottom three quarters of image); within the latter are nests of loosely arranged spindle cells in an organoid distribution (Hematoxylin-and-eosin stain).

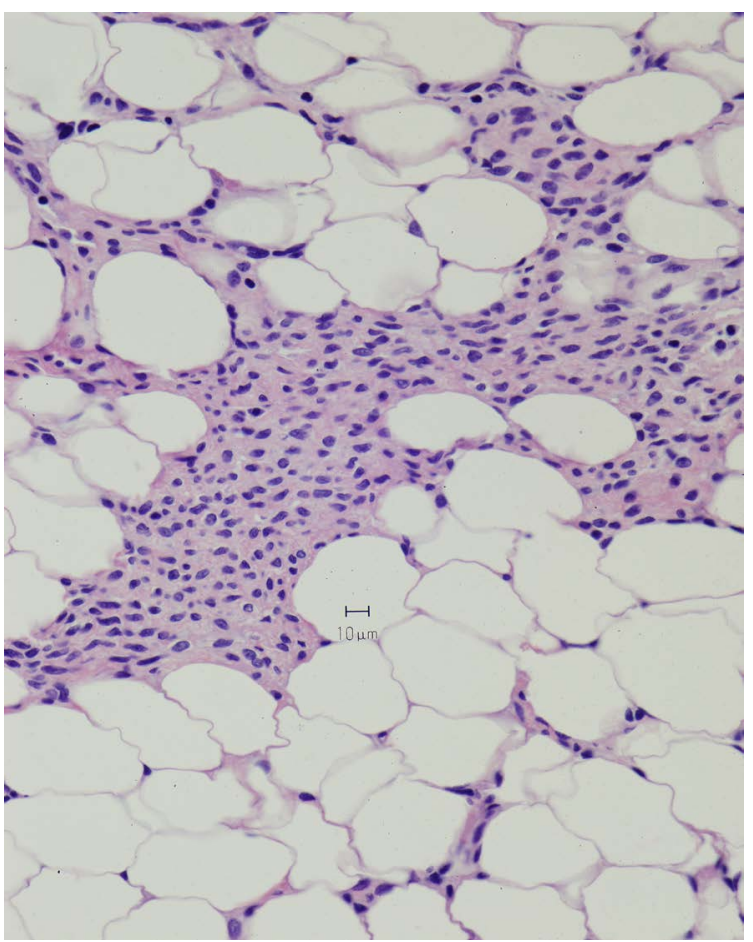

Figure 7. At high magnification, the loosely arranged spindle cells are surrounded by mature adipose tissue and show no significant cytologic atypia and no significant mitotic activity (Hematoxylin-and-eosin stain). 


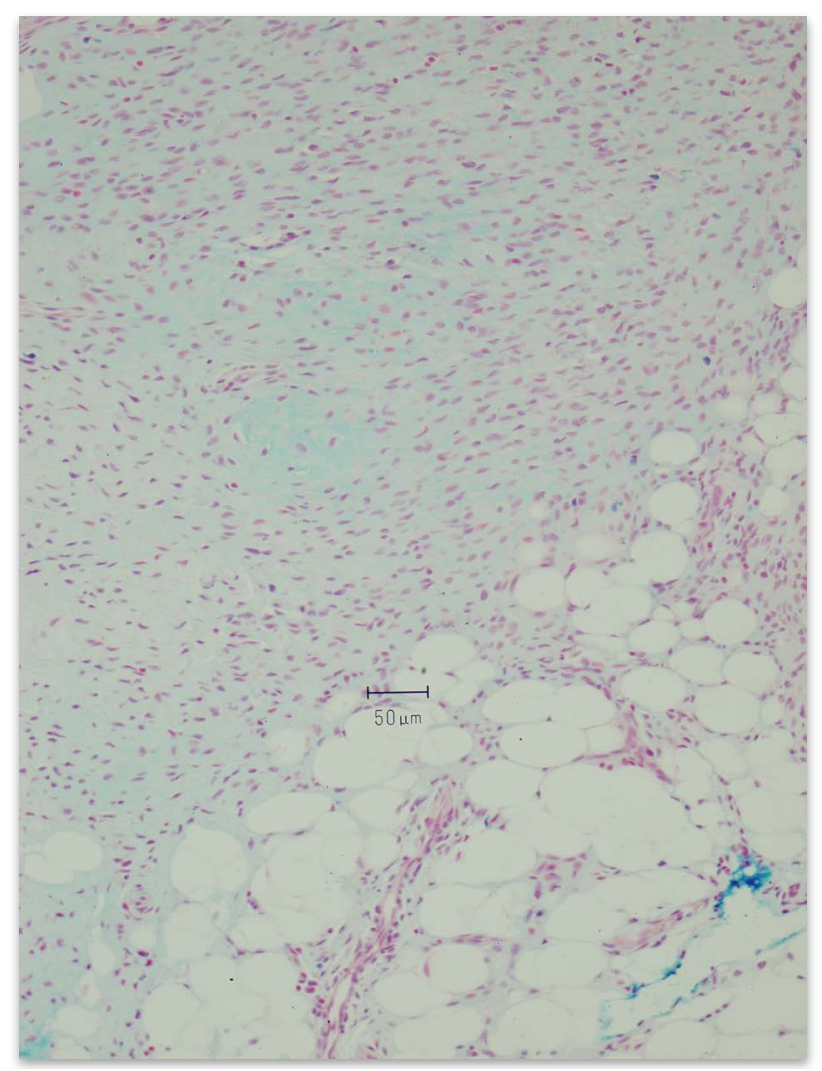

Figure 8. The loosely arranged spindle cells are within an Alcian-blue-positive mucoid matrix (faint blue stain near center of image; the dark blue in the corner of the image is ink used to mark the specimen margin prior to sectioning) (Alcian blue stain).

Upon presentation, the patient was presumptively diagnosed with fibrous tumor of the hand. No incisional biopsy or advanced imaging was acquired because of the simple clinical picture; though, MRI as been utilized to characterize complex HoI at the hand [2] [3] [6]. During surgical exploration, two unique surgical challenges presented themselves: First, the tumor was more adherent to the skin than anticipated; Secondly, the tumor extended more deeply than clinical examination would suggest. There was no plane of dissection between the tumor and the skin. This challenge was overcome by sub-dermal dissection, elevating the skin similarly to a full thickness skin graft procedure. This approach was important in order to completely isolate and remove the tumor but likely also contributed to the post operative dehiscence.

The depth of surgical dissection was greater than anticipated pre-operatively. Meticulous dissection had to be performed in proximity to the superficial palmar arch and the common digital nerves. Reflecting its benign nature, the tumor was non-adherent to the deep tissues, but the close relation to the child's delicate neurovascular bundles required a level of comfort with microsurgical technique to be able to liberate the tumors from the surrounding tissues.

\section{Conclusion}

Though benign in nature, HoI can be a source of significant morbidity when presenting in the hand. Careful surgical technique is required to prevent morbidity and recurrence. The close adherence to the skin can cause some wound healing complications. Adherence to the deep tissues is not observed on this benign tumor; however, microsurgical skills may be required in face of lesions in close proximity to the delicate neurovascular structures in this patient age population. Magnetic resonance imaging may have value for surgical planning in apparently superficial lesions; though, this value must be weighed against the inherent risks of sedation to the patient and the additional cost of advanced imaging. 


\section{References}

[1] Reye, R. (1956) A Consideration of Certain Subdermal Fibrous Tumours of Infancy. Journal of Pathology \& Bacterial, 72, 149-154.

[2] Song, Y., Lee, I. and Kim, H. (2010) Fibrous Hamartoma of Infancy in the Hand: Unusual Location and MR Imaging Findings. Skeletal Radiology, 39, 1035-1038. http://dx.doi.org/10.1007/s00256-010-0896-2

[3] Keskin, M., Tosun, Z., Toy, H., et al. (2007) Subdermal Fibrous Hamartoma of Infancy in the Hand. Scandinavian Journal of Plastic and Reconstructive Surgery and Hand Surgery, 41, 211-214. http://dx.doi.org/10.1080/02844310600723174

[4] Lakshminarayanan, R., Konia, T. and Welborn, J. (2005) Fibrous Hamatoma of Infancy a Case Report with Associated Cytogenetic Findings. Archives of Pathology \& Laboratory Medicine, 129, 520-522.

[5] Jebson, P. and Louis, D. (1997) Fibrous Hamartoma of Infancy in the Hand: A Case Report. Journal of Hand Surgery, 22A, 740-742. http://dx.doi.org/10.1016/S0363-5023(97)80139-6

[6] Netscher, D., Baumholtz, M., Popek, E., et al. (2009) Non-Malignant Fibrosing Tumors in the Pediatric Hand: A Clinicopatholigc Case Review. Hand, 3, 2-11. http://dx.doi.org/10.1007/s11552-008-9148-5

[7] Saab, S., McClain, C. and Coffin, C. (2014) Fibrous Hamatoma of Infancy a Clinicopathologic Analysis of 60 Cases. The American Journal of Surgical Pathology, 38, 394-401. http://dx.doi.org/10.1097/PAS.0000000000000104

[8] Grynspan, D., Meir, K., Senger, C., et al. (2007) Cutaneous Changes in Fibrous Hamartoma of Infancy. Journal of Cutaneous Pathology, 34, 39-43. http://dx.doi.org/10.1111/j.1600-0560.2006.00575.x

[9] Sotelo-Avila, C. and Bale, P.M. (1994) Subdermal Fibrous Hamartoma of Infancy: Pathology of 40 Cases and Differential Diagnosis. Pediatric Pathology, 14, 39-52. http://dx.doi.org/10.3109/15513819409022024 